\title{
La segregación residencial en el México urbano: niveles y patrones
}

\author{
Paavo Monkkonen. Universidad de Hong Kong, Pokfulam, Hong Kong.
}

RESUMEN | Los cambiantes modelos de desarrollo urbano en Latinoamérica han atraído la atención hacia la segregación residencial. Con datos del censo mexicano del año 2000, este artículo describe los patrones espaciales y niveles de segregación por etnicidad y estatus socioeconómico en más de cien ciudades, confirmando que muchos de los que son reconocidos se mantienen en muchas y variadas ciudades. Los hogares con ingresos bajos y empleos informales tienden a vivir en áreas periféricas de la ciudad, mientras que los de ingresos altos y empleados formalmente están más concentrados. Las áreas de ingresos elevados son más densas y socioeconómicamente diversas. Más aún, existe una relación estadísticamente significativa entre segregación y tamaño de ciudad: las ciudades mayores están más segregadas. De manera similar, las diferencias regionales también son grandes y estadísticamente significativas, lo que demuestra la importancia tanto de los períodos históricos de urbanización del país como de los distintos niveles de desarrollo económico regional.

PALABRAS CLAVE | estructura urbana, geografía urbana, segregación

\begin{abstract}
Changingpatterns of urban development in Latin America have drawn increasing attention to residentialsegregation, yet there are no systematic quantitative analyses in theliterature. Using data from the Mexican census of 2000, this paper measures spatial patterns and levels of segregation by ethnicity and socioeconomic status in over one hundred cities. Findings confirm that many recognized patterns hold across a wide variety of cities. Low-income and informally employed households tend to live in peripheral areas of the city, while high-income households are more centrally concentrated. High-income areas are denser and more socioeconomically diverse. Further, the paper shows that there is a statistically significant relationship between segregation by income and city size; larger cities are more segregated. Similarly, regional differences in patterns of segregation are also statistically significant and large, demonstrating the importance of both historical periods of urbanization and levels of regional economic development.
\end{abstract}

KEY WORDS | urban structure, urban geography, segregation 


\section{Introducción}

La segregación residencial de distintos grupos socioeconómicos y de la población indígena en las áreas urbanas de Latinoamérica constituye un fenómeno reconocido y ampliamente discutido, pero esto solo se ha documentado empíricamente de manera reciente, dada la dificultad para acceder a la información necesaria. Entender la segregación en las ciudades de Latinoamérica es importante. Esta región tiene uno de los niveles de desigualdad por ingreso más altos del mundo, así como una población mayoritariamente urbana y pronunciadas divisiones sociales por raza y clase. La localización espacial de estos distintos grupos tiene implicaciones importantes para las relaciones sociales y la gobernabilidad en la región (Roberts \& Wilson, 2009).

El presente estudio mide el nivel y los patrones de segregación residencial de los trabajadores empleados en el sector informal, de los indígenas y de los hogares de ingresos altos y bajos en ciudades mexicanas, mediante la utilización de cuatro de las cinco dimensiones de segregación perfiladas por Massey y Denton (1988): la uniformidad, el agrupamiento (clustering), la centralización y la concentración. A pesar de recientes avances en la medición espacial de la segregación que muestran la uniformidad y el agrupamiento como si fueran la misma medida en dos escalas (Reardon \& Sullivan, 2004), el uso de medidas múltiples sigue siendo útil, dadas las actuales limitaciones de información (sector censal, equivalente al nivel de área geoestadistica básica [AGEB] en lugar de nivel de manzana) y porque al ser convencionales, permiten la comparación entre distintos estudios. Más aún, las medidas de centralización y de concentración describen la localización espacial y la densidad residencial relativa de grupos diferentes, factores que resultan importantes para describir los patrones espaciales y compararlos con los de otros países.

Este estudio representa uno de los primeros análisis de segregación que abarca todo el sistema urbano de un país latinoamericano, contribución importante dada la tendencia a enfocarse en las grandes áreas metropolitanas y la creciente proporción de población que vive en las ciudades medias y más pequeñas (Portes \& Roberts, 2005). Aún más, un análisis nacional resguarda de generalizar al país entero la estructura espacial de algunas ciudades. Finalmente, la comparación de niveles y patrones de segregación en diferentes tipos de ciudades permite la formulación de algunas hipótesis respecto de sus determinantes.

México constituye un buen caso de estudio, por varias razones. Primero, porque posee un gran número de ciudades. En segundo lugar, tiene uno de los niveles más elevados de PIB per cápita en América Latina (Banco Mundial, 2008) y un nivel de desigualdad en el ingreso por encima del promedio de la región (Gasparini, 2003). México también cuenta con una población indígena sustantiva y, a pesar de una política nacionalista que promueve el concepto de mestizaje, o mezcla racial, la discriminación basada en el color de la piel sigue siendo claramente evidente en las oportunidades sociales y de empleo, así como en la representación de dicha población en los medios de comunicación. Finalmente, las comunidades o fraccionamientos 
cerrados (condominios), los barrios bajos (barriadas) urbanos y los asentamientos ilegales — los símbolos visuales de la segregación residencial— son característicos de las áreas urbanas del país.

Los resultados de este estudio confirman y cuantifican algunos de los patrones generalmente aceptados de segregación residencial en las ciudades latinoamericanas: la localización más céntrica de los hogares de ingresos elevados frente a los hogares de bajos ingresos, así como la heterogeneidad en el ingreso de los barrios de ingresos elevados comparados con los de bajos ingresos. Además, la medición de los patrones de segregación proporciona un entendimiento matizado del asentamiento de hogares de bajos ingresos en las periferias urbanas. Aunque los hogares de bajos ingresos tienden a residir en localizaciones más periféricas que los de ingresos más elevados y las áreas periféricas tienen porcentajes elevados de hogares con bajos ingresos, estos últimos realmente se encuentran relativamente muy dispersos en las ciudades mexicanas.

Un resultado sorprendente del estudio es la evidencia de que la mayoría de los hogares de bajos ingresos en el México urbano vive en áreas de la ciudad cuya densidad es relativamente baja, en profundo contraste con la imagen estereotipada de barrios bajos urbanos densos, que se debe en cierta medida a la tendencia de los investigadores urbanos a enfocarse en las grandes áreas metropolitanas. De hecho, los hogares de ingresos altos en las ciudades mexicanas tienen densidades más altas que los hogares de bajos ingresos. Esto es lógico, dada su tendencia a vivir en las partes centrales de la ciudad y la intensidad más alta de desarrollo en tierras localizadas más centralmente.

La segregación de los indígenas sigue patrones similares a aquellos de los hogares de ingresos bajos y de los trabajadores empleados en el sector informal; esto no llama a sorpresa, debido al gran grado de traslape entre estos tres grupos. No obstante, los niveles de segregación de los indígenas son más altos y los patrones son más extremos, lo cual indica niveles aún mayores de discriminación (Flórez, Medina \& Urrea, 2001). Los indígenas ocupan mucho menos espacio por vivienda y viven más periféricamente que los hogares de bajos ingresos o los empleados en el sector informal.

Además de la evidencia de los promedios de los diferentes indicadores de segregación correspondientes al país, el estudio analiza los promedios en ciudades de diferentes tamaños y en regiones diferentes. La variación en los niveles de segregación por ingreso, medidos por desigualdad e índice de entropía, es estadísticamente significativa entre ciudades de tamaños diferentes. Esto encaja con las predicciones de la teoría económica urbana estándar: la mayor diferenciación de los mercados de tierra y de los barrios en ciudades más grandes lleva a mayores niveles de separación de grupos con ingresos diferentes (Mills \& Hamilton, 1994). Sin embargo, lo mismo no es verdadero para los hogares con trabajadores empleados en el sector informal y para los indígenas, lo que sugiere que existen otras fuerzas distintas del ingreso que determinan los patrones de localización residencial de estos dos grupos.

La diferencia en los patrones de segregación a gran escala - agrupamiento, centralización y concentración - entre ciudades de tamaños diferentes no es estadísticamente significativa, aunque en términos generales, lo es entre ciudades de distintas 
regiones. Esto sugiere que los crecientes valores de la tierra y la diferenciación en los mercados del suelo no determinan estos patrones de gran escala de localización residencial, y plantea interrogantes acerca de los factores que los determinan. Entre las posibles explicaciones se encuentran los distintos períodos históricos de desarrollo urbano en las diferentes regiones de México y las disimilitudes en las economías regionales.

Para el desarrollo de estos temas, en la siguiente sección del documento se revisa la literatura empírica sobre segregación residencial en América Latina, con un enfoque en estudios recientes que utilizan métodos comparables. La tercera sección presenta las cuatro series de medidas de segregación. A continuación se utiliza la ciudad de Mérida, Yucatán, para ilustrar visualmente los patrones de segregación en México. El trabajo concluye con un breve resumen.

\section{Revisión de la literatura}

Latinoamérica tiene una historia de segregación residencial sistemática en áreas urbanas que data del período colonial. Un conjunto de leyes españolas, que iniciaron las Leyes de Burgos en 1512 y luego se extendieron como Recopilación de Leyes del Reino de las Indias en 1680, estableció pautas estrictas sobre casi cada aspecto de planeación de la ciudad. Estos códigos buscaron regular las interacciones entre los colonos españoles y los indígenas, al tiempo que prohibían la residencia de grupos indígenas en las áreas habitadas por los españoles y la "residencia de mulatos, mestizos y negros en compañía de los indios” (Mörner \& Gibson, 1962, p. 561).

A pesar de la continua discriminación basada en el color de la piel (Flórez, Medina \& Urrea, 2001), la discusión contemporánea acerca de la segregación urbana en América Latina generalmente se refiere a la segregación socioeconómica, con la excepción notable de Brasil (Telles, 1992). Tal falta de enfoque en la segregación racial proviene principalmente de la política de mestizaje, o mezcla racial, que tiende a dominar el discurso sobre la identidad racial en la región y evita abrir el debate sobre el racismo (Telles, 2007). Esto es especialmente cierto para el caso de México.

Mientras el debate acerca de la conexión entre raza y clase en Estados Unidos persistió durante décadas (Massey \& Denton, 1993), en México solo está empezando a aparecer. La conexión espacial entre raza, clase y pobreza en México también es diferente. La desigualdad entre la localización de viviendas de bajos ingresos y trabajos en Estados Unidos (Ihlanfeldt \& Sjoquist, 1998) se invierte. La vivienda para los grupos de bajos ingresos se encuentra principalmente en la periferia de las ciudades, pero muchos trabajos formales aún no se han suburbanizado (Suárez Lastra \& Delgado Campos, 2007).

También son diferentes en ambas regiones los procesos de ocupación de la ciudad por parte de los sectores de menores ingresos. En Estados Unidos, el incremento en los ingresos durante la segunda mitad del siglo XX, combinado con el desarrollo suburbano basado en el uso del automóvil y la regulación del uso del suelo en las áreas suburbanas, confinó a las minorías pobres y étnicas a la residencia en las 
ciudades centrales (Mieszkowski \& Mills, 1993). En América Latina, en cambio, la urbanización rápida de la última mitad del siglo XX llevó a la proliferación de asentamientos informales de vivienda en la periferia de las ciudades y a su incorporación gradual en el área urbana (Mangin, 1967; Ford, 1996; Ward, 2001).

La estructura espacial de las ciudades latinoamericanas se ha caracterizado tradicionalmente por tres rasgos principales: los grupos de ingresos más bajos ocupan áreas de densidad baja, periféricas y con servicios pobres; los grupos de ingresos elevados se agrupan en un área de la ciudad, generalmente con un vértice en el centro histórico, y se mueven hacia el exterior en una dirección; y existe una mayor homogeneidad socioeconómica en los barrios de bajos ingresos (Griffin \& Ford, 1980; Sabatini, 2003). Y aunque el reciente surgimiento de comunidades cerradas y de desarrollos comerciales en la periferia de las ciudades ha engendrado debates acerca de la suburbanización latinoamericana y la fragmentación del espacio urbano (Ford, 1996; Borsdorf, 2003), la mayoría del espacio periurbano sigue estando habitado principalmente por los grupos de bajos ingresos.

El efecto de los nuevos tipos de desarrollo de vivienda en la periferia urbana en México es diferente al de otros países latinoamericanos. A comienzos de la década de los noventa, ciertos cambios en las políticas habitacionales llevaron a un incremento significativo de créditos para la vivienda, a los cuales solo tenían acceso quienes contaran con un trabajo asalariado (Monkkonen, 2011a). Dicho sistema de financiamiento, junto con las prácticas de construcción de vivienda a gran escala, han producido comunidades cerradas, aunque no necesariamente para las familias de altos ingresos; de hecho, en su mayoría han sido para la clase obrera (García Peralta \& Hoffer, 2006).

La atención académica a la segregación residencial en las ciudades latinoamericanas ha aumentado en años recientes debido a estos cambios y al acceso a datos censales de mayor calidad geográfica (Roberts \& Wilson, 2009). Algunos estudiosos sostienen que las nuevas modalidades de crecimiento urbano están exacerbando la disparidad social y cambiando la escala de segregación en las ciudades latinoamericanas, desde patrones de gran escala a grupos de segregación más pequeños, distribuidos irregularmente (Sabatini, Cáceres \& Cerda, 2001). Este debate acerca de la escala hace más importantes que nunca la consistencia y solidez de las mediciones.

Un volumen recientemente editado (Roberts \& Wilson, 2009) ejemplifica el incremento en el uso de métodos cuantitativos en la investigación sobre segregación en América Latina. Dicho estudio constituye una aplicación de nuevos métodos de análisis espacial, como el indicador local de asociación espacial que describiremos más adelante, que hace uso de la información georreferenciada disponible desde hace poco tiempo. Como otros trabajos recientes (Rodríguez \& Arriagada, 2004; Skop, Peters, Amaral, Potter \& Fusco, 2006; Peters \& Skop, 2007), se trata de un examen detallado de la segregación en algunos estudios de caso sobre ciudades: Santiago de Chile, Ciudad de México, Montevideo, São Paulo en Brasil, Lima en Perú, y Austin, Texas. Estos singulares estudios de caso sobre ciudades son importantes para la comprensión del fenómeno.

En México, una revisión de la literatura realizada por Schteingart (2001) acerca de la división social del espacio urbano explica que, más allá de la investigación 
basada en las metodologías de la Escuela de Chicago (Park, Burgess \& McKenzie, 1925), la investigación urbana en México tradicionalmente ha puesto el acento en las teorías de la marginalidad y en las críticas a dichas teorías, en lugar de hacerlo sobre las nociones de exclusión social. Schteingart también señala que, hasta la década de 1990, el análisis cuantitativo de la segregación en México era limitado, porque no se hallaba disponible la información adecuada. Desde entonces, se han publicado varios trabajos de investigación cuantitativa sobre exclusión social en México (Alegría, 1994; Ariza \& Solís, 2009; Duhau, 2003; Garza, 1999a; González Arrellano \& Villeneuve, 2006; Hernández Gómez, 2001; Rubalcava \& Schteingart, 2000a y 2000b). Estos estudios utilizan técnicas como el análisis factorial para analizar las cinco ciudades más grandes de México: Ciudad de México, Guadalajara, Monterrey, Puebla y Tijuana.

El documento más pertinente para el presente estudio utiliza las cinco dimensiones de segregación de Massey y Denton (1988) para examinar cuatro aspectos del estatus socioeconómico (ingreso, ocupación, estatus migratorio y nivel de escolaridad) en las tres ciudades más grandes de México: Ciudad de México, Guadalajara y Monterrey (Ariza \& Solís, 2009). Los autores ofrecen algunas evidencias importantes para los modelos espaciales de segregación en México adoptados previamente. Sin embargo, restringen su análisis a una comparación entre los hogares con los ingresos más elevados y todos los demás, lo que genera una imagen incompleta de la segregación en dichas ciudades.

Una excepción al predominio de estudios de caso de la segregación en las grandes áreas metropolitanas de América Latina es el trabajo de Telles(1992; 1995) acerca de Brasil de la década de 1990. Sus dos estudios sobre segregación socioeconómica y racial en ciudades brasileñas son los únicos en América Latina que miden cuantitativamente la segregación en un gran número de ciudades en un país. Sin embargo, aunque hasta la fecha es el análisis más extenso sobre esta materia en la región, en él no se examina la dimensión de agrupamiento de la segregación ni se evalúa patrones espaciales que usan medidas de centralización o concentración.

\section{Medición de la segregación en ciudades mexicanas}

El presente estudio mide la segregación de los trabajadores empleados en el sector informal, de grupos indígenas y de hogares de ingreso alto y bajo, mediante cuatro de las cinco dimensiones de segregación propuestas por Massey y Denton (1988) -la uniformidad, el agrupamiento, la centralización y la concentración-, en las 128 áreas urbanas de México cuya población en el año 2000 fue de más de 50.000 habitantes. La quinta dimensión definida por Massey y Denton, la exposición o aislamiento social, no se calcula. A pesar de su denominación, es simplemente otra medida de uniformidad y, a diferencia de los índices de disimilitud y entropía, es sensible a la proporción de la población que pertenece al grupo que se está analizando. Por lo tanto, si hay pocos miembros del grupo, como en el caso de grupos indígenas en muchas ciudades de México, se registra un aislamiento social muy alto, aunque estos grupos de personas estén distribuidos uniformemente por la ciudad. 
Los datos provienen del censo mexicano de población y vivienda del año 2000 (Instituto Nacional de Estadística y Geografía [INEGI], 2000a) y de la cartografía urbana digital del mismo año (INEGI, 2000b). Los cuadros con los datos fueron realizados por el autor en el nivel de área geoestadística básica (AGEB), similar al sector censal. Las AGEB pueden ser mayores que los sectores censales de Estados Unidos —más de 10.000 personas en algunos casos-, pero son principalmente similares en tamaño, con 2.500 personas en cada una en promedio. A diferencia de los sectores censales estadounidenses, las AGEB solo se definen para áreas urbanas y pueblos.

También nos referimos a las 128 áreas urbanas o ciudades cubiertas por este estudio como Áreas Estadísticas Metropolitanas (AEM). En México, el término área metropolitana' ha sido definido recientemente de manera oficial (Secretaría de Desarrollo Social, Consejo Nacional de Población \& Instituto Nacional de Estadística, Geografía e Informática, 2004) como las ciudades que abarcan más de un municipio. Sin embargo, esta definición excluye muchas grandes ciudades que quedan dentro de los límites de un municipio. Así, en el presente estudio, las AEM para el caso de México se definen mediante la utilización de la definición de AEM de Estados Unidos, es decir, el área urbana comprendida dentro de una zona de desplazamiento entre residencia y trabajo alrededor de un centro urbano de 50.000 o más personas.

El estudio emplea variables proxy para empleo informal y etnicidad. La variable proxy para empleo informal se basa en la interpretación existente de trabajo informal (Castells \& Portes, 1989; Biles, 2007) y se compone de tres variables del censo: la situación de seguridad en términos de salud, la ocupación, y la industria donde la persona se encuentra empleada (Suárez Lastra, 2007). La situación de seguridad médica indica empleo formal, ya que todos los trabajadores asalariados y sus familias tienen garantizado por ley el seguro médico por parte del Instituto Mexicano del Seguro Social (Diario Oficial de la Federación, 2006a y 2006b). La ocupación y la industria del empleo también son buenos indicadores; se considera que los profesionales están empleados en el sector formal, al igual que quienes trabajan en la atención a la salud, la educación, las finanzas, las telecomunicaciones u otras industrias administradas por el gobierno. Pese a que existen limitaciones en esta definición de empleo informal, representa en gran medida el estado del arte y capta las distintas dimensiones del fenómeno (Suárez Lastra, 2007).

La variable proxy para etnicidad se refiere a si una persona habla una lengua indígena. Aunque también es una medida imperfecta, ya que excluye a un número importante de personas que padecen discriminación basada en el color de la piel, es la única variable disponible (Telles, 2007).

Los datos de ingreso están en el nivel de hogar. El ingreso en México se mide frecuentemente por múltiplos de salario mínimo recibidos e informados de manera exacta. En el año 2000, el salario mínimo se fijó entre 32,7 y 37,9 pesos mexicanos, o entre 4,13 y 4,79 dólares estadounidenses, por día, cifras cuya variación se debe a las diferencias regionales. En este estudio, los hogares de ingreso elevado se definen como aquellos que percibieron más de cinco salarios mínimos; y los hogares de ingresos bajos, aquellos que percibieron un salario mínimo o menos. Esta clasificación es la 
utilizada por la oficina censal mexicana al momento de reportar las tabulaciones al nivel de sector. Los cinco salarios mínimos establecidos para los grupos de ingresos elevados no constituyen un ingreso muy alto; sin embargo, representan someramente el decil de ingresos más elevados - 11,5\% de los hogares - en una ciudad promedio.

Los datos síntesis y las medidas de segregación informadas aquí se presentan como promedios para todas las ciudades - pequeñas, medias y grandes - y para las ciudades en seis regiones diferentes de México: noroeste, noreste, centro, la región de la Ciudad de México, el sur y la región Pacífico. La Figura 1 es un mapa de México que indica los límites de las seis regiones y la localización de las ciudades pequeñas, medias y grandes. Como allí puede observarse, la región del sur no tiene ninguna ciudad grande, mientras que la región de la Ciudad de México y las regiones del noroeste tienen tres cada una.

Figura 1 | Mapa de las áreas urbanas de México

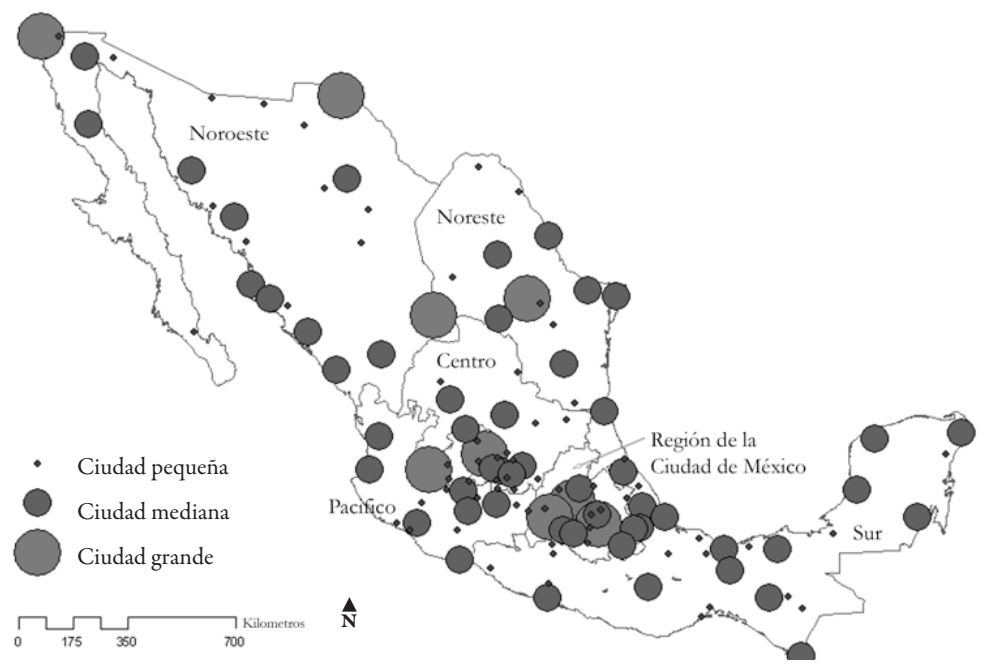

FUente Elaboración del autor con datos del Instituto Nacional de Estadística y Geografía (2000A Y 2000B).

El Cuadro 1 presenta la información resumida para las variables con las que se midió la segregación. Estos promedios proporcionan una idea inicial acerca de qué tan drásticamente difiere la distribución del ingreso en México respecto de Estados Unidos o los países europeos. Los hogares de bajos ingresos exceden en número a los de ingresos elevados en casi cada una de las ciudades, y por dos a uno o más en promedio en las ciudades más pequeñas. Naturalmente, esto alterará la idea de segregación socioeconómica tal y como se concibe tradicionalmente, vale decir, como el aislamiento de una población pequeña de bajos ingresos. 
CUADRO 1 | Proporción de población empleada en el sector informal, de indígenas y de ingresos altos y bajos en 128 ciudades, por tamaño de ciudad y región

\begin{tabular}{|c|c|c|c|c|c|}
\hline $\begin{array}{l}\text { CATEGORÍA DE } \\
\text { CIUDAD }\end{array}$ & $\begin{array}{l}\text { NÚMERO DE } \\
\text { CIUDADES }\end{array}$ & $\begin{array}{l}\text { PORCENTAJE } \\
\text { EN EL } \\
\text { SECTOR } \\
\text { INFORMAL }\end{array}$ & $\begin{array}{l}\text { PORCENTAJE } \\
\text { DE } \\
\text { INDÍGENAS }\end{array}$ & $\begin{array}{l}\text { PORCENTAJE } \\
\text { CON } \\
\text { INGRESOS } \\
\text { ALTOS }\end{array}$ & $\begin{array}{l}\text { PORCENTAJE } \\
\text { CON } \\
\text { INGRESOS } \\
\text { BAJOS }\end{array}$ \\
\hline Todas las ciudades & 128 & 46,8 & 4,1 & 11,5 & 32,9 \\
\hline Ciudades grandes & 9 & 41,4 & 1,6 & 17,0 & 23,5 \\
\hline Ciudades medias & 52 & 42,1 & 3,6 & 12,3 & 31,2 \\
\hline Ciudades pequeñas & 67 & 51,1 & 4,8 & 9,5 & 35,7 \\
\hline $\begin{array}{l}\text { Estadística de F } \\
(2,125)\end{array}$ & NA & $8,1^{* *}$ & 0,4 & $20,3^{* *}$ & $8,3^{* *}$ \\
\hline Noroeste & 24 & 36,3 & 1,2 & 15,2 & 25,0 \\
\hline Noreste & 15 & 32,6 & 0,6 & 13,6 & 26,9 \\
\hline Centro & 19 & 51,1 & 0,6 & 10,6 & 33,0 \\
\hline $\begin{array}{l}\text { Región de la Ciudad } \\
\text { de México }\end{array}$ & 18 & 54,0 & 5,7 & 11,4 & 37,5 \\
\hline Sur & 32 & 50,5 & 10,9 & 9,2 & 39,3 \\
\hline Pacífico & 20 & 53,6 & 1,4 & 9,4 & 30,8 \\
\hline $\begin{array}{l}\text { Estadística de F } \\
(5,122)\end{array}$ & NA & $13,9^{* *}$ & $4,7^{* *}$ & $3,9^{* *}$ & $9,2^{* *}$ \\
\hline
\end{tabular}

FUente Instituto Nacional de Estadística y Geografía, 2000A.

NOTA LAS CIUDADES GRANDES TIENEN MÁS DE UN MILLÓN DE HABITANTES, LAS CIUDADES MEDIAS TIENEN ENTRE 200.000 Y UN MILLÓN DE RESIDENTES Y LAS CIUDADES PEQUEÑAS TIENEN MENOS DE 200.000 HABITANTES. LA Región NOROESTE incluye los estados de Baja California, Baja California Sur, ChiHuahua, Durango, Sinaloa y Sonora; la noreste, Coahuila, Nuevo León y Tamaulipas; el Centro, Aguascalientes, Guanajuato, Querétaro, San Luis Potosí y Zacatecas; la región de la Ciudad de México, el Distrito Federal, Hidalgo, el estado de México, Morelos, Puebla y Tlaxcala; el sur, Campeche, Chiapas, Guerrero, Oaxaca, Quintana Roo, Tabasco, Veracruz y Yucatán; y el Pacífico, Colima, Jalisco, Michoacán y NaYARIT.

${ }^{*} \mathrm{Y}^{* *}$ INDICAN LA SIGNIFICANCIA EN LOS NIVELES 0,05 Y 0,01.

NA $=$ NO APLICA.

Existen diferencias clara y estadísticamente significativas entre las ciudades más grandes y las más pequeñas en todas las variables distintas de aquella que muestra la proporción de población indígena. Se utilizó un análisis de varianza (ANOVA) de un sentido para probar diferencias y todas fueron significativas (las estadísticas de F se reportan en el cuadro), excepto las correspondientes a la proporción de población indígena. La variación en el ingreso es especialmente alta: las ciudades más grandes tienen en promedio casi dos veces la proporción de hogares con ingresos elevados que las ciudades pequeñas. 
De manera similar, la variación regional es amplia y las diferencias en todas las variables son estadísticamente significativas. Hay proporciones mayores de hogares con ingresos altos y trabajadores empleados en el sector formal en el norte del país, mientras las ciudades del sur tienen una porción mayor de indígenas, y son más pobres. Esto coincide con las tendencias generales de desarrollo regional en México, como son el desarrollo industrial de las ciudades del centro del país de principios y mediados del siglo, la reciente industrialización de las ciudades norteñas y la persistente pobreza en el sur (Garza, 1999b).

\section{Uniformidad}

Los índices de disimilitud y de entropía se usan para medir la uniformidad, la dimensión de segregación más comúnmente medida, la cual representa la diferencia en la distribución de grupos en subunidades de una ciudad. El índice de disimilitud se usa porque es la medida más común de uniformidad. Puede entenderse como el porcentaje de un grupo dado que necesitaría reubicarse para alcanzar una distribución uniforme de los grupos.

Recientemente se ha comprobado que el índice de entropía constituye el índice de uniformidad más apropiado (Reardon \& Firebaugh, 2002). Indica hasta qué punto la composición de los sectores se desvía de una variable dada a lo largo y ancho de la ciudad, y puede interpretarse como la diferencia porcentual entre la distribución existente de grupos dados y una distribución completamente uniforme. Adicionalmente, a diferencia del índice de disimilitud, el índice de entropía puede utilizarse para calcular la segregación entre muchos grupos; en este caso, cinco categorías de ingreso de los hogares. Los valores para los índices de disimilitud y entropía se presentan en el Cuadro 2.

A pesar de que algunas ciudades de México tienen niveles altos de segregación de grupos indígenas, el valor promedio para el índice de disimilitud $(0,32)$ es más bajo que los niveles encontrados en Estados Unidos para grupos de afroamericanos o latinos (0,64 y 0,51, respectivamente) en el año 2000 (Iceland, Weinberg \& Steinmetz, 2002), y más bajo que los índices de minorías étnicas en la mayoría de las ciudades europeas para las que existe información comparable (Musterd, 2005). Este fenómeno se puede explicar recurriendo a varias razones. Por ejemplo, el proceso de urbanización paulatino en México, combinado con el sistema de suelo de uso colectivo - es decir, el ejido-, pudiera contribuir a niveles bajos de segregación. Esto es, un ejido compuesto por personas indígenas en la periferia de una ciudad que se urbaniza en un proceso de venta de lotes a migrantes no indígenas podría resultar en la formación de una colonia relativamente mixta.

Los hogares de ingresos altos poseen un valor mucho más elevado, tanto en el índice de disimilitud como en el índice de entropía, que los propios de los hogares con empleos informales y de los hogares de ingresos bajos. Los valores bajos para los últimos dos grupos indican que se distribuyen de manera relativamente uniforme en los sectores censales, y ello en parte porque representan proporciones significativas de la población. El valor promedio de los niveles de segregación de los hogares de bajos in- 
gresos de México es similar a los del Reino Unido o de Holanda, cuyos valores van de 0,15 a 0,25 (Musterd, 2005). Es menor que el de otras ciudades de América Latina para las que existe información disponible. Por ejemplo, Santiago de Chile tuvo un índice de disimilitud entre 0,30 y 0,47 (Rodríguez \& Arriagada, 2004), y Lima, Perú, tuvo un índice agregado de disimilitud de segregación socioeconómica de 0,52 (Peters \& Skop, 2007). En Estados Unidos, el índice de disimilitud promedio para los hogares de bajos ingresos en cien ciudades fue 0,36, más del doble del de México (Musterd, 2005).

CUADRO 2 | Valores de los índices de disimilitud y de entropía en 128 ciudades, por tamaño de ciudad y región

\begin{tabular}{|c|c|c|c|c|c|c|c|c|c|}
\hline \multirow[b]{2}{*}{$\begin{array}{l}\text { Categoría de } \\
\text { ciudad }\end{array}$} & \multicolumn{4}{|c|}{ ÍNDICE DE DISIMILITUD } & \multicolumn{5}{|c|}{ ÍNDICE DE ENTROPÍA } \\
\hline & Informal & Indígenas & $\begin{array}{l}\text { Ingreso } \\
\text { alto }\end{array}$ & $\begin{array}{c}\text { Ingreso } \\
\text { bajo }\end{array}$ & Informal & Indígenas & $\begin{array}{l}\text { Ingreso } \\
\text { alto }\end{array}$ & $\begin{array}{c}\text { Ingreso } \\
\text { bajo }\end{array}$ & $\begin{array}{l}\text { Ingreso (5 } \\
\text { categorías) }\end{array}$ \\
\hline $\begin{array}{l}\text { Todas las } \\
\text { ciudades }\end{array}$ & 0,19 & 0,32 & 0,31 & 0,17 & 0,04 & 0,10 & 0,09 & 0,03 & 0,04 \\
\hline $\begin{array}{l}\text { Ciudades } \\
\text { grandes }\end{array}$ & 0,19 & 0,41 & 0,35 & 0,18 & 0,05 & 0,15 & 0,13 & 0,04 & 0,06 \\
\hline $\begin{array}{l}\text { Ciudades } \\
\text { medias }\end{array}$ & 0,20 & 0,34 & 0,34 & 0,17 & 0,05 & 0,11 & 0,11 & 0,04 & 0,05 \\
\hline $\begin{array}{l}\text { Ciudades } \\
\text { pequeñas }\end{array}$ & 0,19 & 0,32 & 0,29 & 0,16 & 0,05 & 0,09 & 0,07 & 0,04 & 0,04 \\
\hline $\begin{array}{l}\text { Estadística de } \\
\mathrm{F}(2,125)\end{array}$ & 0,95 & 0,56 & $13,17^{* *}$ & $3,44^{*}$ & 0,25 & 1,02 & $29,06^{* *}$ & 0,28 & $31,91^{* *}$ \\
\hline Noroeste & 0,16 & 0,32 & 0,31 & 0,17 & 0,03 & 0,08 & 0,09 & 0,03 & 0,05 \\
\hline Noreste & 0,16 & 0,37 & 0,33 & 0,16 & 0,03 & 0,08 & 0,11 & 0,03 & 0,05 \\
\hline Centro & 0,20 & 0,29 & 0,30 & 0,17 & 0,05 & 0,05 & 0,09 & 0,04 & 0,04 \\
\hline $\begin{array}{l}\text { Región de la } \\
\text { Ciudad de } \\
\text { México }\end{array}$ & 0,22 & 0,42 & 0,34 & 0,18 & 0,06 & 0,19 & 0,10 & 0,04 & 0,04 \\
\hline Sur & 0,21 & 0,30 & 0,33 & 0,18 & 0,06 & 0,11 & 0,10 & 0,04 & 0,05 \\
\hline Pacífico & 0,19 & 0,37 & 0,28 & 0,14 & 0,05 & 0,11 & 0,07 & 0,03 & 0,03 \\
\hline $\begin{array}{l}\text { Estadística de } \\
\mathrm{F}(5,122)\end{array}$ & $9,08^{* *}$ & 0,73 & $2,41^{*}$ & $3,04^{*}$ & $9,21^{* *}$ & $3,07^{*}$ & 2,17 & $3,59^{* *}$ & $2,31^{*}$ \\
\hline
\end{tabular}

FUENTE Elaboración del autor con datos del Instituto NaCional de Estadística y Geografía, 2000A Y 2000B.

NOTA LAS CIUDADES GRANDES TIENEN MÁS DE UN MILLÓN DE HABITANTES, LAS CIUDADES MEDIAS TIENEN ENTRE 200.000 Y UN MILLÓN DE RESIDENTES Y LAS CIUDADES PEQUEÑAS TIENEN MENOS DE 200.000 HABITANTES. LA REGIÓN NORoeste incluye los estados de Baja California, Baja California Sur, Chihuahua, Durango, Sinaloa y Sonora; la noreste, Coahuila, Nuevo León y Tamaulipas; el centro, Aguascalientes, Guanajuato, QueRétaro, San Luis Potosí y Zacatecas; la Región de la CiUdad de México, el Distrito Federal, Hidalgo,

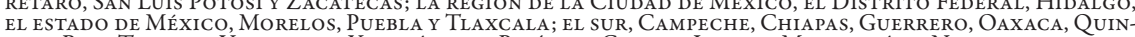
tana Roo, Tabasco, Veracruz y Yucatán; y el Pacífico, Colima, Jalisco, Michoacán y Nayarit.

${ }^{*} \mathrm{Y}{ }^{* *}$ INDICAN LA SIGNIFICANCIA EN LOS NIVELES 0,05 Y 0,01. 
El Cuadro 2 también muestra la estadística F de un ANOVA de un sentido utilizado para probar la significancia en la diferencia entre los índices de disimilitud y entropía para las diferentes categorías de tamaño de ciudad y las diferentes regiones. La significancia estadística de las diferencias entre niveles de segregación por ingreso es mucho más fuerte en ciudades de distinto tamaño que en regiones diferentes. Esto es lógico, dadas las predicciones de la teoría económica urbana presentadas en la introducción. Las ciudades más grandes tienen niveles más altos de disimilitud tanto de los hogares de ingresos altos como de los de ingresos bajos, y de los cinco índices de entropía por grupo de ingresos. La situación es completamente diferente para los hogares empleados en el sector informal y los hogares indígenas. No hay ninguna diferencia significativa en los niveles de segregación de los hogares con empleo informal en las ciudades de diferente tamaño, y la de los hogares indígenas es mínima.

\section{Agrupamiento}

El agrupamiento se refiere al nivel de proximidad entre unidades territoriales donde residen las categorías similares de población, e indica si en su distribución se autocorrelacionan entre sí, presentando un patrón no aleatorio. Como se mencionó anteriormente, esto también puede concebirse como una extensión de la medida de uniformidad a una escala mayor. Debido a las limitaciones de la información, aquí utilizamos una medida distinta. El índice global de Moran (I) es una prueba de autocorrelación espacial que indica si los sectores con proporciones altas de un grupo se localizan cerca de otros sectores con proporciones elevadas de ese grupo (Moran, 1950). En este caso, se usa tanto para medir si hay un grado estadísticamente significativo de agrupamiento en una ciudad y a qué nivel. El índice de Moran (I) es la medida de agrupamiento más comúnmente utilizada en la investigación sobre segregación en América Latina (Roberts \& Wilson, 2009).

Los valores del índice de Moran (I) para todas las ciudades de México que tienen un nivel de agrupamiento estadísticamente significativo se presentan en las columnas dos a cinco del Cuadro 3, y el porcentaje de ciudades que tienen un nivel significativo de agrupamiento se reporta en las columnas seis a nueve. Los valores mayores del índice de Moran (I) indican un grado más alto de autocorrelación espacial, donde los valores negativos muestran la independencia espacial y los positivos indican una autocorrelación espacial perfecta.

El Cuadro 3 también muestra las estadísticas de F para un ANOVA de un sentido, utilizadas para probar si las diferencias entre los valores promedio del índice de Moran (I) son estadísticamente significativas en ciudades de diferente tamaño y en regiones diferentes. La variación en el valor del índice de Moran (I) por tamaño de ciudad no es estadísticamente significativa para cualquiera de las variables medidas, a pesar de las grandes diferencias en los valores promedio en algunos casos, pero las diferencias regionales sí lo son.

No se encuentra disponible un valor promedio de agrupamiento medido con el índice de Moran (I) para las áreas metropolitanas de otro país, aunque el análisis de varias ciudades desarrollado por Martin (1991) sugiere que el agrupamiento por ingreso es ligeramente más alto en Estados Unidos (alrededor de 0,40), y el agrupamiento por 
raza puede ser mucho más alto (una cifra de 0,80 en la ciudad de Oklahoma). Existe evidencia limitada según la cual otras ciudades latinoamericanas también poseen niveles elevados de agrupamiento: Lima, Perú, tuvo un índice de Moran (I) de 0,70 por estatus socioeconómico (Peters \& Skop, 2007), superior al de la inmensa mayoría de las ciudades de México.

CUADRo 3 | Valores del índice de Moran (I) en 128 ciudades, por tamaño de ciudad y región

\begin{tabular}{|c|c|c|c|c|c|c|c|c|}
\hline \multirow[b]{2}{*}{$\begin{array}{l}\text { Categoría de } \\
\text { ciudad }\end{array}$} & \multicolumn{4}{|c|}{ VALORES DEL ÍNDICE DE MORAN (I) } & \multicolumn{4}{|c|}{$\begin{array}{c}\text { PROPORCIÓN DE CIUDADES CON } \\
\text { VALORES SIGNIFICATIVOS }\end{array}$} \\
\hline & Informal & Indígenas & $\begin{array}{l}\text { Ingreso } \\
\text { alto }\end{array}$ & $\begin{array}{l}\text { Ingreso } \\
\text { bajo }\end{array}$ & Informal & Indígenas & $\begin{array}{l}\text { Ingreso } \\
\text { alto }\end{array}$ & $\begin{array}{l}\text { Ingreso } \\
\text { bajo }\end{array}$ \\
\hline $\begin{array}{l}\text { Todas las } \\
\text { ciudades }\end{array}$ & 0,48 & 0,34 & 0,38 & 0,38 & 0,80 & 0,38 & 0,65 & 0,79 \\
\hline $\begin{array}{l}\text { Ciudades } \\
\text { grandes }\end{array}$ & 0,53 & 0,23 & 0,47 & 0,32 & 1,00 & 0,78 & 0,89 & 0,89 \\
\hline $\begin{array}{l}\text { Ciudades } \\
\text { medias }\end{array}$ & 0,45 & 0,28 & 0,37 & 0,34 & 0,60 & 0,35 & 0,54 & 0,60 \\
\hline $\begin{array}{l}\text { Ciudades } \\
\text { pequeñas }\end{array}$ & 0,41 & 0,28 & 0,31 & 0,39 & 0,94 & 0,34 & 0,70 & 0,93 \\
\hline $\begin{array}{l}\text { Estadística de F } \\
(2,125)\end{array}$ & 2,23 & 1,06 & 0,33 & 1,65 & NA & NA & NA & NA \\
\hline Noroeste & 0,47 & 0,17 & 0,36 & 0,25 & 0,79 & 0,17 & 0,58 & 0,88 \\
\hline Noreste & 0,30 & 0,50 & 0,39 & 0,22 & 0,80 & 0,40 & 0,67 & 0,87 \\
\hline Centro & 0,41 & 0,16 & 0,31 & 0,48 & 0,63 & 0,26 & 0,58 & 0,63 \\
\hline $\begin{array}{l}\text { Región de la } \\
\text { Ciudad de } \\
\text { México }\end{array}$ & 0,77 & 0,51 & 0,65 & 0,69 & 0,83 & 0,44 & 0,61 & 0,72 \\
\hline Sur & 0,46 & 0,31 & 0,33 & 0,43 & 0,94 & 0,63 & 0,84 & 0,91 \\
\hline Pacífico & 0,44 & 0,31 & 0,30 & 0,33 & 0,70 & 0,20 & 0,45 & 0,60 \\
\hline $\begin{array}{l}\text { Estadística de F } \\
(5,122)\end{array}$ & $3,74^{* *}$ & 0,78 & $2,77^{*}$ & $3,22^{* *}$ & NA & NA & NA & NA \\
\hline
\end{tabular}

FUente Elaboración del autor con datos del Instituto NaCional de Estadística y Geografía, 2000A Y 2000B.

Nota: LAS CIUDAdES GRANDES TIENEN MÁS DE UN MILLÓN DE HABITANTES, LAS CIUDADES MEDIAS TIENEN ENTRE 200.000 Y UN MILLÓN DE RESIDENTES Y LAS CIUDADES PEOUEÑAS TIENEN MENOS DE 200.000 HABITANTES. LA REGIÓN NOROESTE INCluye LOS ESTADos De Baja California, Baja California Sur, Chihuahua, Durango, Sinaloay Sonora; la noreste, Coahuila, Nuevo León y Tamaulipas; el Centro, Aguascalientes, Guanajuato, Querétaro, San Luis Potosí y Zacatecas; la Región de la Ciudad de México, el Distrito Federal, Hidalgo, el estado de México, Morelos, Puebla y Tlaxcala; el sur, Campeche, Chiapas, Guerrero, OAXaca, Quintana Roo, Tabasco, Veracruz y Yucatán; y el Pacífico, Colima, Jalisco, Michoacán y Nayarit.

${ }^{*} \mathrm{Y}^{* *}$ INDICAN LA SIGNIFICANCIA EN LOS NIVELES 0,05 Y 0,01 .

$\mathrm{NA}=$ NO APLICA 


\section{Centralización}

Un índice de centralización absoluto (ACI, por sus siglas en inglés) se calcula para medir el grado en que los diferentes grupos viven cerca del centro de la ciudad. El ACI valora el rango de uno negativo a uno, con valores más altos que indican un nivel mayor de centralización. Un valor de uno negativo indica descentralización extrema, con todos los miembros de un grupo localizados en los sectores periféricos; un valor de cero significa que todos los miembros están uniformemente distribuidos del centro a la periferia; y un valor de uno, que todos los miembros de ese grupo viven en el polígono central.

CUADRO 4 | Índice de centralización absoluta y valores de Delta en 128 ciudades, por tamaño de ciudad y región

\begin{tabular}{|c|c|c|c|c|c|c|c|c|}
\hline \multirow[b]{2}{*}{ Categoría de ciudad } & \multicolumn{4}{|c|}{$\begin{array}{c}\text { ÍNDICE DE CENTRALIZACIÓN } \\
\text { ABSOLUTA }\end{array}$} & \multicolumn{4}{|c|}{ DELTA } \\
\hline & Informal & Indígenas & $\begin{array}{l}\text { Ingreso } \\
\text { alto }\end{array}$ & $\begin{array}{l}\text { Ingreso } \\
\text { bajo }\end{array}$ & Informal & Indígenas & $\begin{array}{l}\text { Ingreso } \\
\text { alto }\end{array}$ & $\begin{array}{l}\text { Ingreso } \\
\text { bajo }\end{array}$ \\
\hline Todas las ciudades & 0,21 & 0,18 & 0,37 & 0,20 & 0,37 & 0,46 & 0,48 & 0,37 \\
\hline Ciudades grandes & 0,21 & 0,16 & 0,38 & 0,22 & 0,38 & 0,49 & 0,50 & 0,39 \\
\hline Ciudades medias & 0,18 & 0,17 & 0,35 & 0,19 & 0,36 & 0,47 & 0,48 & 0,37 \\
\hline Ciudades pequeñas & 0,23 & 0,19 & 0,39 & 0,21 & 0,37 & 0,44 & 0,47 & 0,36 \\
\hline Estadística de $\mathrm{F}(2,125)$ & 1,74 & 0,17 & 0,83 & 0,76 & 0,12 & 1,58 & 0,49 & 0,43 \\
\hline Noroeste & 0,12 & 0,06 & 0,21 & 0,13 & 0,32 & 0,41 & 0,39 & 0,34 \\
\hline Noreste & 0,25 & 0,28 & 0,40 & 0,24 & 0,42 & 0,52 & 0,50 & 0,43 \\
\hline Centro & 0,25 & 0,29 & 0,38 & 0,24 & 0,37 & 0,44 & 0,47 & 0,37 \\
\hline $\begin{array}{l}\text { Región de la Ciudad de } \\
\text { México }\end{array}$ & 0,21 & 0,13 & 0,50 & 0,21 & 0,37 & 0,49 & 0,56 & 0,37 \\
\hline Sur & 0,21 & 0,17 & 0,41 & 0,20 & 0,36 & 0,44 & 0,50 & 0,36 \\
\hline Pacífico & 0,24 & 0,19 & 0,39 & 0,22 & 0,39 & 0,48 & 0,47 & 0,37 \\
\hline Estadística de $\mathrm{F}(5,122)$ & $3,99^{* *}$ & $2,94^{*}$ & $13,94^{* *}$ & $2,86^{*}$ & $4,79^{* *}$ & $3,47^{* *}$ & $10,38^{* *}$ & $2,86^{*}$ \\
\hline
\end{tabular}

FUENTE Elaboración del autor con datos del Instituto Nacional de Estadística y Geografía, 2000A Y 2000B.

NOTA LAS CIUDADES GRANDES TIENEN MÁS DE UN MILLÓN DE HABITANTES, LAS CIUDADES MEDIAS TIENEN ENTRE 200.000 Y UN MILLÓN DE RESIDENTES Y LAS CIUDADES PEQUEÑAS TIENEN MENOS DE 200.000 HABITANTES. LA REGIÓN NOROESTe incluye los estados de Baja California, Baja California Sur, Chihuahua, Durango, Sinaloa y Sonora; la noreste, Coahuila, Nuevo León y TAmaulipas; El Centro, Aguascalientes, Guanajuato, Querétaro, San Luis Potosí y Zacatecas; la Región de la Ciudad de México, el Distrito Federal, Hidalgo, el estado de México, Morelos, Puebla y Tlaxcala; el sur, Campeche, Chiapas, Guerrero, Oaxaca, Quintana Roo, Tabasco, Veracruz y Yucatán; y el Pacífico, Colima, Jalisco, Michoacán y Nayarit.

${ }^{*} \mathrm{Y}^{* *}$ INDICAN LA SIGNIFICANCIA EN LOS NIVELES 0,05 Y 0,01. 
Los valores del ACI para todas las ciudades de México se presentan en el Cuadro 4, al igual que las estadísticas de F para un ANOVA de un sentido utilizadas para probar las diferencias en los valores promedio en ciudades de distinto tamaño y en regiones diferentes. El ACI medio, correspondiente a los empleados en el sector informal, es de 0,21 y el de los grupos de bajos ingresos es de 0,20. Al comparar estos valores con los de la población total, que es de 0,25 , resulta evidente que los empleados en el sector informal y las poblaciones de bajos ingresos son más propensos a vivir en las áreas periféricas. En contraste, los hogares de ingresos altos tienen un $\mathrm{ACI}$ promedio de 0,37 , lo que indica que viven mucho más centralmente que la población en conjunto. Otra manera de plantear este contraste es que, en promedio, en las 128 ciudades consideradas en este estudio, casi el 70\% de los hogares con ingresos elevados vive en el $10 \%$ interior de la superficie de una ciudad, mientras que solo lo hace el $55 \%$ de los hogares con ingresos bajos.

Existe poca variación en el ACI en las ciudades de tamaños diferentes. Esta consistencia sugiere que la centralización no responde a los cambios en los valores de la tierra, lo que lleva a pensar en homogeneidad en el proceso de desarrollo urbano en todas las ciudades. Sin embargo, regionalmente existen diferencias significativas en los valores del ACI, sobre todo entre las ciudades del noroeste y el noreste. Esto podría derivarse del desarrollo más reciente de ciudades en el noroeste y, como consecuencia, de la diferencia en la estructura urbana (Alegría, 1994; Garza, 1999b).

\section{Concentración}

Para medir la superficie de tierra o la densidad residencial de las áreas ocupadas por los diferentes grupos en las ciudades mexicanas, se utiliza el índice Delta. El índice Delta puede interpretarse de manera similar a la disimilitud, como el porcentaje de población de un grupo que tendría que cambiar de residencia para alcanzar una densidad uniforme en toda la ciudad. Así, cuanto más alto sea el índice Delta, menor es la superficie de tierra ocupada por un grupo. Los valores de Delta se presentan en el Cuadro 4. En promedio, los hogares empleados en el sector informal y los hogares de bajos ingresos están ligeramente menos concentrados que la población en general, mientras que los hogares de ingresos elevados y los indígenas están mucho más concentrados que la población en general. Es sorprendente que la población indígena tenga valores tan elevados de Delta, ya que en otros índices tienden a tener valores similares a los que poseen los hogares de ingresos bajos.

El hecho de que grupos empleados en el sector informal y aquellos de bajos ingresos parezcan ocupar más tierra que sus contrapartes empleadas en el sector formal y que los que poseen ingresos altos, no quiere decir que ocupen espacios de vida más amplios; más bien, simplemente indica que viven en áreas con menor densidad promedio. Frecuentemente, estas áreas son de baja densidad porque se encuentran en las laderas de las montañas o en otras áreas en las que el desarrollo de alta densidad no es posible, o porque recientemente se establecieron en áreas que todavía no han sido completamente urbanizadas.

$\mathrm{Al}$ igual que con las medidas de agrupamiento y centralización, la diferencia en los valores del índice Delta en las ciudades de diferente tamaño no es significativa, 
mientras que las diferencias regionales sí lo son. Las ciudades del noroeste de México se destacan por tener valores inusualmente bajos del índice Delta, lo que indica densidades más bajas y niveles menores de concentración para todos los grupos, aunque las diferencias relativas entre los grupos son similares. La estructura de la ciudad en México parece depender más de las diferencias regionales que del tamaño.

\section{Visualización de los patrones de segregación}

Una representación visual de los patrones espaciales de segregación de México se presenta mediante la utilización de la ciudad de Mérida, Yucatán. Mérida es una de las áreas metropolitanas medianas que está creciendo, y se escogió como referencia, ya que está altamente segregada y muestra patrones ejemplares de segregación. La Figura 2 muestra la distribución espacial de los hogares de bajos ingresos por AGEB en Mérida, y un segundo coropleta ${ }^{1}$ señala aquellas $A G E B$ que tienen autocorrelación espacial estadísticamente significativa de acuerdo con la prueba local de Moran (I). El índice de Moran (I) local ofrece una perspectiva matizada del agrupamiento al probar si la autocorrelación espacial entre un sector y su vecindario es estadísticamente significativa (Anselin, 1995). Los sectores que forman los grupos pueden identificarse en un mapa y ofrecen una representación visual útil de los patrones de segregación.

El patrón ejemplificado por la Figura 2 es la tendencia de los hogares de bajos ingresos a agruparse en grupos pequeños y fragmentados, lo que también vale para los trabajadores empleados en el sector informal y los indígenas, mientras que los trabajadores empleados en el sector formal y los hogares de ingresos elevados se agrupan en un área grande, localizada centralmente. En Mérida, por ejemplo, hay un solo grupo significativo de hogares de ingresos altos, mientras que existen siete grupos separados de hogares de ingresos bajos. La Figura 2 también ilustra las mayores proporciones de personas de bajos ingresos empleadas en el sector informal e indígenas que se encuentran en los sectores periféricos.

Los patrones de agrupamiento ilustrados en la Figura 2 son similares al tercer patrón de segregación discutido en la introducción, es decir, que las áreas de ingresos elevados tienden a tener mayor heterogeneidad en el ingreso. Al utilizar el índice local de Moran (I), es posible probar estadísticamente esta aseveración comparando la diversidad de ingresos en un sector tal y como se midió por la entropía. Así, se calculó una puntuación de entropía para cada AGEB, cálculo en el que un resultado mayor indica un mayor nivel de heterogeneidad del ingreso. Entonces, la puntuación promedio de la entropía en los grupos de los vecindarios de ingresos elevados se puede comparar con la entropía de los grupos de bajos ingresos. Esto se realizó en las 101 ciudades que tienen grupos significativos tanto de grupos de ingresos altos como de grupos de ingresos bajos, y se detectó que la entropía de los grupos de ingreso elevado es de 1,55, mientras que la de las áreas de bajos ingresos es de 1,22. La entropía de los vecindarios de ingresos altos excede la de los barrios de bajos ingresos por 0,33 , lo que representa aproximadamente $20 \%$ de sus entropías combinadas y una diferencia que es estadísticamente significativa en el nivel 0,01, según una prueba $\mathrm{T}$ de dos muestras con varianzas desiguales $(\mathrm{t}=8.89)$.

1 Un mapa en que los colores de las diferentes sub áreas corresponden al valor de una variable 


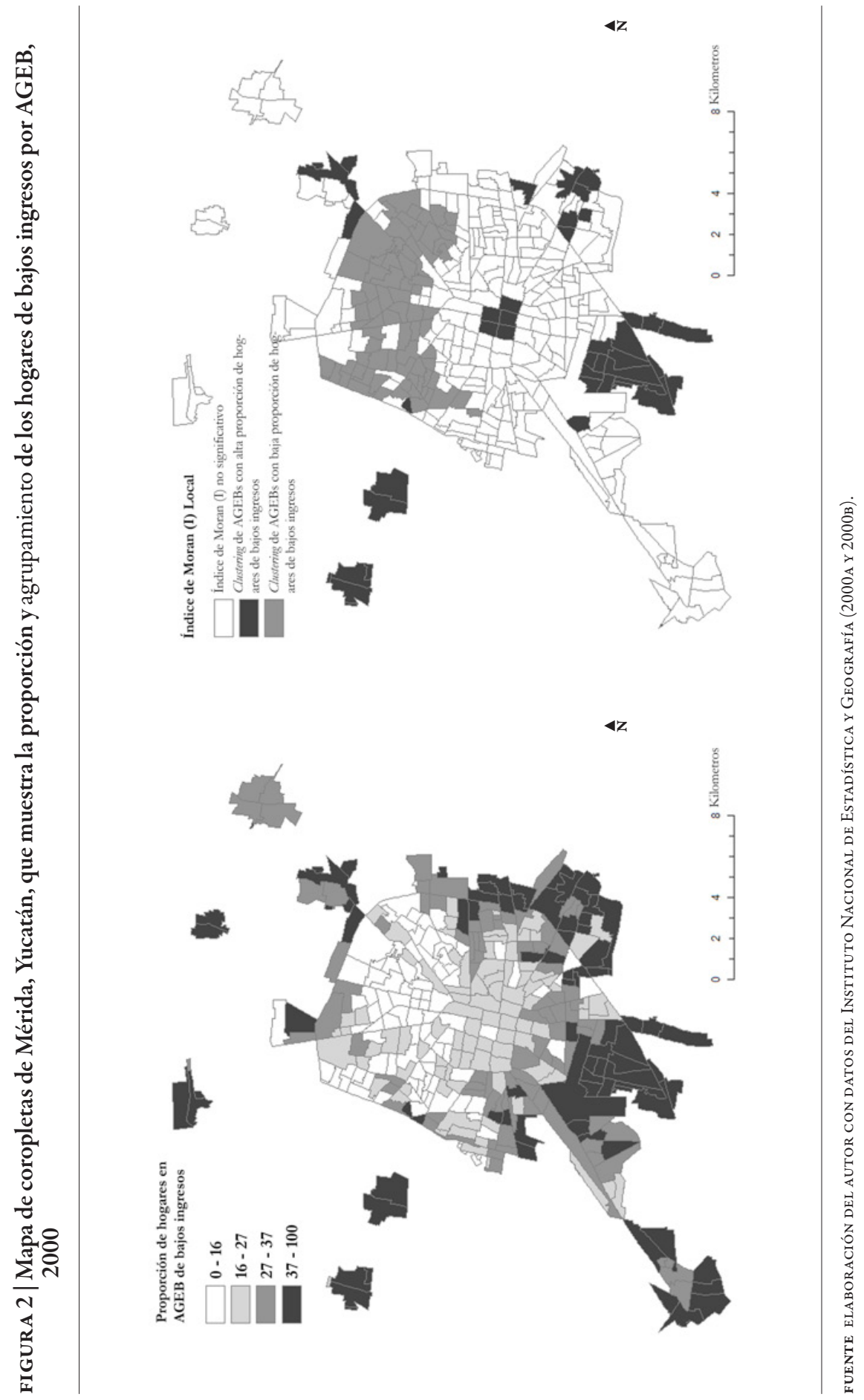




\section{Conclusiones}

El presente estudio ofrece un análisis comprensivo de la segregación residencial en el sistema urbano de un país latinoamericano. La segregación de los trabajadores empleados en el sector informal, de los indígenas y de los hogares de ingresos altos y bajos se midió en las 128 áreas metropolitanas de México. Se analizaron las magnitudes y los patrones espaciales de segregación con mediciones de cuatro dimensiones de segregación y la referencia visual de una ciudad altamente segregada, Mérida, Yucatán. El documento proporciona evidencia concreta para dos de las tendencias de segregación que caracterizan las ciudades en América Latina: el agrupamiento de los grupos de ingresos elevados en una zona central y la mayor heterogeneidad del ingreso en las áreas de ingresos altos comparada con las de residentes de ingresos bajos. Además, se desarrolla una interpretación matizada de una tercera tendencia asumida comúnmente, esto es, la dispersión de los grupos de bajos ingresos a lo largo de las áreas periféricas. Finalmente, el documento muestra que la segregación de los hogares de bajos ingresos y de las minorías étnicas no es tan elevada comparada con la de Estados Unidos o Europa.

Los niveles relativamente bajos de segregación socioeconómica en México probablemente se explican por una combinación de factores, incluidas las condiciones de la tierra y de los mercados de vivienda, la estructura de gobierno local y la naturaleza de la regulación del uso del suelo y su (falta de) aplicación. Hasta hace poco, la mayoría de los hogares de México accedía a la vivienda por medio de un proceso de autoconstrucción, en el que las familias construyen sus viviendas lentamente a lo largo del tiempo. Este proceso lleva a una porción grande de los hogares de bajos ingresos a instalarse en la periferia urbana. Tanto el limitado desarrollo especulativo de la vivienda suburbana y la incapacidad de los proyectos habitacionales suburbanos para incorporarse a las ciudades a medida que estas reducen la posibilidad de suburbanización para los ingresos medios y altos, como la mínima aplicación de la regulación de uso del suelo y de construcción, significan que los hogares a menudo mejoran sus viviendas actuales en lugar de mudarse a una vivienda nueva. Así, los niveles de segregación se mantienen relativamente bajos.

Sin embargo, las nuevas políticas de vivienda y la expansión del financiamiento de vivienda en México han generado un cambio importante en la forma de urbanización en el país. La mayor parte del desarrollo de viviendas ocurre en nuevas colonias extensas construidas por compañías privadas y adquiridas con créditos del Instituto del Fondo Nacional de la Vivienda para los Trabajadores (Infonavit). Los nuevos proyectos habitacionales crean una forma de urbanización con más infraestructura que la irregular, pero son más homogéneos y con una densidad más alta (Monkkonen, 2011b). Asimismo, esta nueva forma de urbanización afecta los niveles de segregación socioeconómica. Un estudio sobre los cambios en los niveles de segregación en el sistema urbano de México de 1990 a 2000 por Monkkonen (2012) encuentra que en ciudades con más construcción de nuevos conjuntos habitacionales, el nivel de segregación socioeconómica es más alto. No obstante, que- 
dan muchas preguntas sobre la naturaleza y la escala de la relación entre la nueva forma de desarrollo urbano y la segregación socioespacial.

La importancia de los mercados de suelo urbano y el uso del suelo en la consecuente segregación también se ha convertido recientemente en un tema de investigación empírica en Estados Unidos (Galster \& Cutsinger 2007). Incluso la naturaleza del acceso a la vivienda, que depende en mucho de las características del desarrollo urbano, es especialmente importante en el estudio de la segregación socioeconómica. El hecho de que la variación en los niveles de segregación de los diferentes grupos de ingreso sea significativa en ciudades de tamaño diferente en México encaja con las predicciones de la teoría económica urbana estándar. Se esperaba una variación similar en los patrones de segregación en ciudades de tamaños diferentes, pero no se encontró.

En este análisis, los niveles significativos de variación regional en los patrones de segregación apoyan aún más la noción de que los procesos históricos de desarrollo urbano son patrones determinantes de segregación más importantes que los factores del mercado universal de suelos. Evidentemente, esta explicación necesita un desarrollo más profundo antes de que pueda alcanzarse una conclusión contundente.

\section{Referencias bibliográficas}

Alegría, T. (1994). Segregación socioespacial urbana. El ejemplo de Tijuana. Estudios Demográficos y Urbanos, 9(2), 411-437.

Anselin, L. (1995). Local indicators of spatial association. Geographic Analysis, 27, 93-115.

Ariza, M. \& Solís, P. (2009). Dinámica socioeconómica y segregación espacial en tres áreas metropolitanas de México, 1990 y 2000. Estudios Sociológicos, 27(79), 171-209.

Biles, J. J. (2009). Informal work in Latin America. Competing perspectives and recent debates. Geography Compass, 3(1), 214-236. doi 10.1111/j.1749-8198.2008.00188.x.

Borsdorf, A. (2003). Cómo modelar el desarrollo y la dinámica de la ciudad latinoamericana. EURE, 29(86), 37-49. doi: 10.4067/S0250-71612003008600002.

Castells, M. \& Portes, P. (1989). World underneath The origins, dynamics and effects of the informal economy. En A. Portes, M. Castells \& L. A. Benton (Eds.), The informal economy. Studies in advanced and less developed countries (pp. 11-33). Baltimore, MD: Johns Hopkins University Press.

Diario Oficial de la Federación (2006a). Ley Federal de Trabajo. México: Secretaría de Gobernación.

Diario Oficial de la Federación (2006b). Ley del Seguro Social. México: Secretaría de Gobernación.

Duhau, E. (2003). División social del espacio metropolitano y movilidad residencial. Papeles de Población [Universidad Autónoma del Estado de México], 9(36), 161-210.

Flórez, C. E., Medina, C. \& Urrea, F. (2001). Understanding the cost of social exclusion due to race or ethnic background in Latin America and Caribbean countries. [Documento inédito]. Washington, DC: Banco Interamericano de Desarrollo.

Ford, L. R. (1996). A new and improved model of Latin American city structure. Geographical Review, $86(3), 437-440$. 
Galster, G. \& Cutsinger, J. (2007). Racial settlement and metropolitan land-use patterns. Does sprawl abet black-white segregation? Urban Geography, 28(6), 516-553.

Gasparini, L. (2003). Income inequality in Latin America and the Caribbean. Evidence from household surveys. Documento de Trabajo n. ${ }^{\circ}$ 2, Centro de Estudios Distributivos, Laborales y Sociales (Cedlas), Universidad Nacional de la Plata, Argentina. Publicado en Económica (2005), 51(1-2), 29-57, disponible en http://econpapers.repec.org/article/lapjournl/542.htm.

García Peralta, B. \& Hoffer, A. (2006). Housing for the working class on the periphery of Mexico City. A new version of gated communities. Social Justice, 33(3), 105-120.

Garza, G. (1999a). La estructura socio-espacial de Monterrey, 1970-1990. Estudios Demográficos y Urbanos, n. ${ }^{\circ} 14,545-597$.

Garza, G. (1999b). Global economy, metropolitan dynamics, and urban policies in Mexico. Cities, 16(3), 149-170. http://dx.doi.org/10.1016/S0264-2751(99)00013-X.

González Arrellano, S. \& Villeneuve, P. (2006). Transformaciones en el espacio socio-residencial de Monterrey, 1990-2000. Estudios Demográficos y Urbanos [El Colegio de México, A.C.], 22(1), 143-178.

Griffin, E. \& Ford, L. (1980). A model of Latin American city structure. Geographical Review, 70(4), $397-422$.

Hernández Gómez, E. (2001). Globalización y segregación urbana en Tijuana, Baja California. Comercio Exterior, 51(3), 234-242.

Iceland, J., Weinberg, D. H. \& Steinmetz, E. (2002). Racial and ethnic residential segregation in the United States: 1980-2000. Documento de la Oficina del Censo de Estados Unidos presentado en la Annual Meetings of the Population Association of America, mayo 9-11, 2002, Atlanta, GA.

Ihlanfeldt, K. R. \& Sjoquist, D. J. (1998). The spatial mismatch hypothesis. A review of recent studies and their implications for welfare reform. Housing Policy Debate, 9(4), 849-892.

Instituto Nacional de Estadística y Geografía (INEGI), México. (2000a). XII Censo General de Población y Vivienda. Aguascalientes, México: Autor.

Instituto Nacional de Estadística y Geografía (INEGI), México. (2000b). Cartografía Urbana Geoestadistica. Aguascalientes, México: Autor.

Mangin, W. (1967). Latin American squatter settlements. A problem and a solution. Latin American Research Review, 2(3), 65-98.

Marti, D. B. (1991). Geographic Information Systems and their socioeconomic applications. Andover, Hants, Inglaterra: Routledge, Capman, and Hall.

Massey, D. S. \& Denton, N. A. (1988). The dimensions of racial segregation. Social Forces, 67(2), 281-315.

Massey, D. S. \& Denton, N. A. (1993). American apartheid. Segregation and the making of the underclass. Cambridge, MA: Harvard University Press.

Mieszkowski, P. \& Mills, E. S. (1993). The causes of metropolitan suburbanization. Journal of Economic Perspectives, 7(3), 135-147.

Mills, E. \& Hamilton, B. (1994). Urban Economics (5a ed.). Nueva York, NY: HarperCollins College Publishers.

Moran, P. A. P. (1950). Notes on continuous stochastic phenomena. Biometrika, 37(1-2), 17-33. doi:10.1093/biomet/37.1-2.17. 
Mörner, M. \& Gibson, C. (1962). Diego Muñoz Camargo and the segregation policy of the Spanish Crown. The Hispanic American Historical Review, 42(4), 558-568.

Monkkonen, P. 2011a. The housing transition in Mexico. Expanding access to housing finance. Urban Affairs Review, 47(11), 672-695.

Monkkonen, P. 2011b. Do Mexican cities sprawl? Housing finance reform and changing patterns of urban growth. Urban Geography, 32(3), 406-423.

Monkkonen, P. 2012. Housing finance reform and increasing socioeconomic segregation in Mexico. International Journal of Urban and Regional Research, disponible en http://dx.doi. org/10.1111/j.1468-2427.2011.01085.x.

Musterd, S. (2005). Social and ethnic segregation in Europe. Levels, causes, and effects. Journal of Urban Affairs, 27(3), 331-348.

Park, R. E., Burgess, E. \& McKenzie, R. (1925). The City. Chicago, IL: University of Chicago Press.

Peters, P. A. \& Skop, E. H. (2007). Socio-spatial segregation in Metropolitan Lima, Peru. Journal of Latin American Geography, 6(1), 150-170.

Portes, A. \& Roberts, B. (2005). The free market city. Latin American urbanization in the years of the neoliberal experiment. Studies in Comparative National Development, 40(1), 43-82. doi: $10.1007 /$ BF02686288.

Reardon, S. F. \& O’Sullivan, D. (2004). Measures of spatial segregation. Sociological Methodology, 34(1), 121-162.

Reardon, S. F. \& Firebaugh, G. (2002). Measures of multigroup segregation. Sociological Research, 32(1), 33-67.

Roberts, B. R. \& Wilson, R. H. (Eds.) (2009). Urban segregation and governance in the Americas. Nueva York: Palgrave-MacMillan.

Rodríguez, J. \& Arriagada, C. (2004). Segregación residencial en la ciudad latinoamericana. EURE, $30(89), 5-24$.

Rubalcava, R. M. \& Schteingart, M. (2000a). Segregación socioespacial. En G. Garza (Ed.), La Ciudad de México en el fin del segundo milenio (pp. 287-296). México: Gobierno del Distrito Federal y El Colegio de México.

Rubalcava, R. M. \& Schteingart, M. (2000b). La división social del espacio en las grandes metrópolis mexicanas. Un estudio comparativo. El mercado de valores (México: Nacional Financiera), $60(4), 20-33$.

Sabatini, F., Cáceres, G. \& Cerda, J. (2001). Residential segregation pattern changes in main Chilean cities. Scale shifts and increasing malignancy. Documento presentado en el Seminario Internacional sobre Segregación en la Ciudad, del Lincoln Institute of Land Policy, julio 26-28, Cambridge, MA.

Sabatini, F. (2003). The social spatial segregation in the cities of Latin America. Documento de Estrategia para el Desarrollo Social. Washington, DC.: Banco Interamericano de Desarrollo.

Schteingart, M. (2001). La división social del espacio en las ciudades. Perfiles Latinoamericanos, $10(19), 13-31$.

Secretaría de Desarrollo Social (Sedesol), Consejo Nacional de Población (Conapo) e Instituto Nacional de Estadística, Geografía e Informática (INEGI). (2004). Delimitación de las Zonas Metropolitanas de México. México: Autores. 
Skop, E., Peters, P. A., Amaral, E., Potter, J., \& Fusco, W. (2006). Chain migration and residential segregation of internal migrants in the metropolitan area of São Paulo, Brazil. Urban Geography, 27(5), 397-421.

Suárez Lastra, M. (2007). Mercados de trabajo y localización residencial en la Zona Metropolitana de la Ciudad de México. Tesis doctoral, Universidad Nacional Autónoma de México, México, DF.

Suárez Lastra, M. \& Delgado Campos, J. (2007). Estructura y eficiencia urbanas. Accesibilidad a empleos, localización residencial e ingreso en la ZMCM 1990-2000. Economía Sociedad y Territorio, 6(23), 693-724.

Telles, E. E. (1992). Residential segregation by skin color in Brazil. American Sociological Review, 57(2), 186-198.

Telles, E. E. (1995). Structural sources of socioeconomic segregation in Brazilian metropolitan areas. The American Journal of Sociology, 100(5), 1199-1223.

Telles, E. E. (2007). Race and ethnicity and Latin America's United Nations Millennium Development Goals. California Center for Population Research, On-Line Working Paper Series 048-07.

Ward, P. M. (2001). The rehabilitation of consolidated irregular settlements in Latin American cities. Documento presentado en el Taller ESF, Coping with Informality and Illegality in Human Settlements, mayo 23-26, Bruselas, Bélgica.

World Bank (2008). World Development Indicators. Washington, DC: Autor. 